\section{UTILIZACIÓN DE HERRAMIENTAS WEB 2.0 EN LA FÍSICA Y QUÍMICA DE BACHILLERATO}

\author{
Felipe Quintanal Pérez \\ Departamento de Ciencias y Tecnología, \\ Colegio marista "La Inmaculada", \\ c/ Sócrates 8 (Granada) \\ felipeqp@maristasmediterranea.com
}

\section{USING WEB 2.0 TOOLS IN HIGH SCHOOL PHYSICS AND CHEMISTRY}

\begin{abstract}
The action research project developed is to link the study of physics of $7^{\text {th }}$ High Scool with different situations that appear in science fiction films, as well as using different Web 2.0 applicatio$n s$ and putting them together in a virtual platform microblogging, Edmodo, as a means of communication and distribution of the work done by students.
\end{abstract}

KEY WORDS: Physics; High School; science fiction; Edmodo; Web 2.0 tools.

\section{INTRODUCCIÓN}

Es notorio señalar que uno de los principales problemas que afrontan actualmente la Didáctica de la Ciencias, el aprendizaje y la enseñanza de la Ciencia escolar se asienta en las actitudes poco interesadas hacia la Ciencia por parte de los alumnos.

Según Quintanal (2011), "este diagnóstico negativo tiene, además, un perfil temporal variable, caracterizado por un punto de inflexión muy notable en la adolescencia temprana según sugieren diversos estudios. Esta progresiva falta de interés de los adolescentes y, especialmente de las chicas, los va alejando de la ciencia escolar, de modo que la consecuencia natural es el abandono de los jóvenes de la ciencia y de las carreras científicas en las primeras elecciones de estudios y carreras, un resultado que preocupa actualmente".

Por otro lado, según Sigalés, Mominó, Meneses y Badía (2008), los diversos informes publicados en el último decenio revelan datos puramente tecnológicos sobre la integración de las TIC en la escuela, pero se muestran ineficaces a la hora de aportar información sobre dicha integración en la actividad formativa y educativa de la escuela, así
RESUMEN: El proyecto de investigación-acción desarrollado trata de enlazar el estudio de la Fisica de $1 .{ }^{\circ}$ de Bachillerato con diversas situaciones que aparecen en las películas de ciencia ficción, así como el empleo de diferentes aplicaciones Web 2.0 y su puesta en común en una plataforma virtual de microblogging, Edmodo, como medio de comunicación y distribución de los trabajos efectuados por los estudiantes.

PALABRAS CLAVE: Física; Bachillerato; ciencia ficción; Edmodo; Herramientas Web 2.0.

como escasos para dirigir las acciones políticas y sociales encaminadas a remozar los obstáculos que ralentizan la implementación de las TIC en la escuela.

La tecnología per se no es una solución, sino que su valor radica en saber utilizarla y aprovechar las facilidades que brinda para la mejora y optimización de los procesos de enseñanza-aprendizaje de los estudiantes. En este contexto, coincidiendo con Beatriz, Álvarez, Dans, Paur y Gallego (2011), la alfabetización científico-tecnológica es la herramienta necesaria para generar conocimientos y competencias, que permitan a cada estudiante vincularse con el resto de su grupo como microexpresión del mundo y, a la vez, poseer cierta autonomía en la toma de decisiones que refleje su idiosincrasia en las formas organizativas de trabajo y en la gestión del conocimiento.

\section{Marco teórico}

La red de redes que es Internet hay que contemplarla hoy en día como un fenómeno que ha evolucionado dinámicamente a través del tiempo hasta llegar al concepto 
actual de Web 2.0. Este concepto se inició en 2004 en las conferencias de la editorial O'Reilly como contraposición a los desarrollos web previos a la explosión de la burbuja tecnológica de 2001. No obstante, se debe considerar que el fenómeno Web 2.0 no anula a la Web 1.0, sino que se fundamenta en ésta y la mejora, completándola. En este sentido, la etiqueta 2.0 implica un dinamismo de la Red que implica la construcción y el desarrollo de una Web social, la cual continúa evolucionando hacia el despliegue de la Web semántica o Web 3.0.

Antes de delimitar el concepto de Web 2.0, es interesante enumerar los principios constitutivos de ésta según las consideraciones de O'Reilly (2005): la Web como plataforma, la inteligencia colectiva, la gestión de la base de datos, la consideración del software como un servicio, el establecimiento de modelos de programación ligera, la instauración del software como servicio integral y la experiencia fructífera del usuario.

Una vez establecido el basamento de estos principios constitutivos se puede presentar una noción de Web 2.0. Al ser un fenómeno poliédrico, aparecen multitud de definiciones, pero, en esta investigación, puede ser útil el concepto de De Clerq (2009): "La Web 2.0 es una nueva generación de servicios y aplicaciones web en línea que facilitan la publicación, el compartir y la difusión de contenidos digitales que fomentan la colaboración y la interacción en línea y que ofrecen unos instrumentos que facilitan la búsqueda y la organización de la información en línea".

Por consiguiente, incorporar las herramientas Web 2.0 en los procesos informativos y formativos, como expresa la plataforma Scopeo, 2009, exige integrar nuevos estilos de comunicación, nuevos roles, nuevas formas de intervención, una amplia panoplia de actividades, es decir, propone una gama variada de retos y desafíos educativos, lo que conlleva a asumir un cambio social y educativo en este caso.

No obstante, estas aplicaciones tecnológicas no están acompañadas de ningún manual de instrucciones o de pautas para generar una acción formativa, por lo que deben ser los educadores, formadores y las instituciones educativas quienes han de idearlas, crearlas, aplicarlas y evaluarlas. Entre los códigos comunes a la acción pedagógica de la Web 2.0 se debe partir de una serie de criterios generales antes de sugerir una acción educativa específica.
Estos criterios pueden ser: Concordancia con la finalidad educativa y/o competencia a desarrollar, conexión con el nivel educativo y los aprendizajes previos de los estudiantes, confirmación de la calidad curricular de los materiales digitales de la asignatura, anuencia de las actividades diseñadas con la competencia digital de los estudiantes, certificación del acceso y disponibilidad de las herramientas Web 2.0, moderación de la función pedagógica de las herramientas Web 2.0 y eficacia sociocultural con el entorno y los participantes.

Las herramientas de las Web 2.0 serán significativas en educación, por lo que puede inferirse de los criterios expuestos anteriormente, cuando respondan a metas de aprendizaje concebidas en base a la interacción, garantizando la posibilidad de mejorar el aprendizaje en red.

Podemos clasificar las rutas educativas de la Web 2.0, a pesar de la constante evolución, innovación y complejidad de su universo, en:

- Redes sociales: Conjunto de herramientas diseñadas para la creación y gestión de comunidades virtuales. En estas redes virtuales sociales los usuarios pueden comunicarse de forma real y efectiva, intercambiando todo tipo de información útil a los miembros de la red. El uso educativo de estas redes se apoya en un enfoque cooperativo del trabajo y en un uso temático. Como ejemplos conocidos se pueden señalar Facebook, Twitter, Tuenti o Ning.

- Crear recursos: Familia de servicios Web 2.0 que permite al grupo de usuarios construir contenidos para ser compartidos y divulgados, apoyando de esta forma el desarrollo de la inteligencia colectiva, pues sirven para crear contenidos de forma descentralizada. Ejemplos muy utilizados son la elaboración de wikis, de blogs o de presentaciones alojadas en Scribd.

- Compartir recursos: Agrupación de servicios de la Web 2.0 que permite a los usuarios compartir recursos multimedia a través de plataformas especializadas. Así, se pueden almacenar y distribuir imágenes, fotografías, vídeos, presentaciones, archivos de audio, etc. Un buen número de experiencias en las aulas sugieren su utilidad a la hora de integrar recursos telemáticos aplicados a la educación en aquellas situaciones donde es necesario publicar y compartir ficheros digitales. Ejemplos muy conocidos son YouTube, Flickr o SlideShare. 
- Recuperar información: Servicios de la Web 2.0 para organizar, a través del filtrado, recomendación o etiquetado, recursos a partir de las necesidades informativas del usuario, permitiendo un acceso selectivo a los contenidos de la Web, así como su distribución masiva. Por tanto, en educación, estas herramientas tecnológicas permiten la búsqueda y acceso a materiales académicos complementarios y proporcionan una infraestructura compartida de recursos, lo que a su vez, puede sustentar redes sociales constituidas en torno al trabajo colaborativo de selección de recursos. Ejemplos destacables son Delicious, Google académico o Technorati.

Se puede concluir, como Quintanal, 2010, que con este panorama tan rico que ofrece la Web 2.0, el aprendizaje colaborativo actual nace y responde a un nuevo contexto sociocultural, donde se define claramente cómo aprendemos (socialmente) y dónde aprendemos (en Red).

\section{Objetivos}

- Aumentar la motivación e incentivar los procesos de enseñanza-aprendizaje de los alumnos para el estudio de la materia de Física y Química de 1. ${ }^{\circ}$ de Bachillerato.

- Ayudar al alumno a descubrir que es el responsable de su propio aprendizaje y que entre ellos se puede generar conocimiento.

- Potenciar el uso de herramientas Web 2.0 como medio eficaz a la hora de realizar diversas tareas y de servir como eje comunicador y solidario entre alumnos y alumnos-profesor, permitiendo la alfabetización digital como eje transversal de la asignatura.

- Implicar al alumnado en el desarrollo de proyectos de investigación-acción desde estrategias dinámicas.

- Colaborar en el descubrimiento de la relación existente entre Ciencia, Sociedad, TIC y vida cotidiana.

\section{Metodología y descripción del PROYeCto}

La experiencia educativa efectuada se ha realizado durante el curso 2010-2011 y se ha dirigido a alumnos de $1 .^{\circ}$ de Bachillerato de las modalidades de Ciencias de la Salud y Tecnología, es decir, que cursaban la asignatura de Física y Química. El número de alumnos que se implicó en el proyecto fue de 46 estudiantes, 22 de Ciencias de la Salud y 24 de Tecnología.

Se detectó durante las primeras semanas del curso una cierta apatía, dejadez y dificultad en el tratamiento de la materia, lo que nos condujo a un replanteamiento de las estrategias didácticas empleadas hasta ese momento.

Dadas las limitaciones tecnológicas en el planteamiento de la asignatura y las propias del centro se pensó proponer un proyecto en el cual las herramientas informáticas tuvieran un valor considerable. Con ello se pretendia favorecer la alfabetización digital, incrementar la autoestima y motivación de los estudiantes, mejorar sus procesos de enseñanza-aprendizaje, desarrollar la comunicación y desarrollar el sentido estético y artístico de los alumnos.

Después del diálogo con los alumnos se seleccionó el universo de las películas de ciencia ficción como temática de su agrado. Se propuso que los trabajos de investigación de los alumnos empleasen diferentes herramientas de la Web 2.0. Después de explicar brevemente cuáles eran las más importantes, se optó por utilizar aquéllas que permitian compartir y crear recursos. Se escogieron, por su solidez y facilidad a la hora de su manejo, las siguientes aplicaciones: BigHugeLabs, Issuu, MyStudiyo, Glog/Glogster y YouTube.

Se propuso, además, emplear otra dimensión de la Web 2.0, las redes sociales. Después de un intercambio de opiniones se concluyó que las tareas debían estar recogidas en una plataforma virtual que permitiese la comunicación entre los estudiantes y entre éstos y el profesor, así como que se pudiesen compartir y visualizar los trabajos de los demás estudiantes, que fuese de fácil manejo y, a ser posible, gratuita. La escogida fue Edmodo.

El siguiente paso lo constituyó la fase inicial del proyecto que consistió en la formación de los equipos de trabajo (parejas), la elección de la película que iba a ser el eje de su trabajo, la realización de una ficha elaborada por los estudiantes que contuviese, al menos, cinco errores físicos o científicos detectados en la película escogida y la corrección de dicha ficha por parte de un experto externo (cf. Figura 1). Se ha de aclarar que se podían analizar diversas secuencias de la película o ésta en su totalidad, 
que las películas debian estar disponibles en YouTube y que experto externo se consideraba a cualquier persona de su ámbito, que no fuese alumno o profesor del centro, con los conocimientos adecuados para poder efectuar la revisión de la ficha realizada. Los trabajos efectuados se recogieron en un $C D$.

FICHA DOS: TABLA DEL EXPERTO

\begin{tabular}{|c|c|c|c|c|}
\hline TIEMPO & $\begin{array}{l}\text { CAMPO DE LA } \\
\text { FÍSICA }\end{array}$ & DESCRIPCIÓN & REALIDAD & LEYES FÍSICAS \\
\hline \multirow[t]{2}{*}{$\begin{array}{l}\text { Durante la mayoría del } \\
\text { vídeo }\end{array}$} & Óptica & Láseres en el espacio & $\begin{array}{l}\text { En el espacio no existe materia, por tanto } \\
\text { la luz coherente no dejaría rastro alguno. }\end{array}$ & Óptica \\
\hline & & & $\begin{array}{l}\text { Sólo debería de verse el impacto sobre } \\
\text { la nave. }\end{array}$ & \\
\hline \multirow[t]{2}{*}{$0: 37,0: 55,1: 03,2: 33$, etc. } & \multirow[t]{2}{*}{ Gravitación } & \multirow{2}{*}{$\begin{array}{l}\text { Hay una serie de disparos } \\
\text { desde el exterior de la nave } \\
\text { hacia el interior, por lo } \\
\text { que produce huecos en el } \\
\text { casco de la nave. Pero esto } \\
\text { sólo produce una pequeña } \\
\text { explosión. }\end{array}$} & \multirow{2}{*}{$\begin{array}{l}\text { Cuando el casco de la nave es } \\
\text { atravesado, el interior de la nave se } \\
\text { comunica con el espacio exterior, por lo } \\
\text { que debería de haber una descompresión } \\
\text { en el interior de la nave. Esto no se ve } \\
\text { hasta el minuto } 2: 42, \text { y antes de este } \\
\text { minuto, se han producido más impactos }\end{array}$} & $\begin{array}{l}\text { Ley de la } \\
\text { Gravitación } \\
\text { Universal }\end{array}$ \\
\hline & & & & $\begin{array}{l}\text { TEORÍA DE } \\
\text { LOS VASOS } \\
\text { COMUNICANTES }\end{array}$ \\
\hline $\begin{array}{l}\text { 0:00 (y durante la mayoria } \\
\text { del vídeo) }\end{array}$ & $\begin{array}{l}\text { Física } \\
\text { Médica }\end{array}$ & $\begin{array}{l}\text { Tippón, es mitad humana, } \\
\text { mitad alien, tiene rasgos } \\
\text { humanos y alienígenas. }\end{array}$ & $\begin{array}{l}\text { Es mitad humano, mitad vulcaniana. } \\
\text { Si ni siquiera es posible un cruce entre } \\
\text { humano y simio, uno entre humano y } \\
\text { alien es inconcebible. }\end{array}$ & $\begin{array}{l}\text { Compatibilidad } \\
\text { genética }\end{array}$ \\
\hline $2: 35,3: 05$, etc. & Fisica Nuclear & $\begin{array}{l}\text { Se produce un disparo a la } \\
\text { nave, y ésta produce una } \\
\text { explosión hacia el exterior. }\end{array}$ & $\begin{array}{l}\text { No puede haber explosiones ni llamas } \\
\text { en el espacio debido a la ausencia de } \\
\text { oxígeno. }\end{array}$ & $\begin{array}{l}\text { Teoria de la } \\
\text { combustión }\end{array}$ \\
\hline \multirow[t]{2}{*}{ Todo el vídeo (ej: 0:00) } & \multirow[t]{2}{*}{ Gravitación } & \multirow[t]{2}{*}{$\begin{array}{l}\text { En la nave, hay una gravedad } \\
\text { equivalente a la de la Tierra. }\end{array}$} & $\begin{array}{l}\text { No puede haber gravedad dentro de la } \\
\text { nave, si no está dentro de un campo } \\
\text { gravitatorio de algún planeta. }\end{array}$ & \multirow[t]{2}{*}{$\begin{array}{l}\text { Ley de la } \\
\text { Gravitación } \\
\text { Universal }\end{array}$} \\
\hline & & & $\begin{array}{l}\text { Sólo puede haber gravedad artificial } \\
\text { dentro de una nave mediante el giro de } \\
\text { la misma alrededor de un eje. Y en el } \\
\text { video, no está girando. }\end{array}$ & \\
\hline $0: 32,3: 05$, etc. & Acústica & $\begin{array}{l}\text { Después de cada disparo, } \\
\text { se oye el sonido del disparo } \\
\text { chocando contra la nave }\end{array}$ & $\begin{array}{l}\text { No es posible que haya sonido alguno en } \\
\text { el espacio, ya que el sonido requiere aire } \\
\text { para viajar, y en el espacio no hay aire. }\end{array}$ & $\begin{array}{l}\text { Ley de la } \\
\text { transferencia } \\
\text { sónica }\end{array}$ \\
\hline
\end{tabular}

Figura 1. Modelo de tabla de errores con la corrección del experto externo para la película Star Trek Enterprise.

Se continuó con la fase de desarrollo del proyecto. Ésta consistió en la elaboración de una presentación multimedia que explicitase los errores detectados en forma de cuestionario de opción múltiple; la conversión y alojamiento de esta presentación como revista digital en Issuu; la elaboración de un póster cinematográfico personalizado de la película escogida y el envío de estas tareas a la plataforma Edmodo, en la cual previamente se habían dado de alta en el grupo correspondiente.

El proyecto se concluyó con la fase final. Esta etapa del proyecto de investigación-acción constó de la siguientes tareas: 
la transformación del cuestionario de la fase anterior en una aplicación multimedia empleando MyStudiyo (cf. Figura 2); la elaboración de una presentación que contuviese, como mínimo, 5 enunciados de problemas basados en la película, así como sus resoluciones; la generación de un mural multimedia que sirviese de presentación del trabajo efectuado utilizando Glog/Glogster; la realización de un experimento casero, relacionado con la película, que pudiera alojarse en la plataforma o ser subido a YouTube, así como un guión que explicase dicho experimento y, por último, la respuesta al cuestionario de autoevaluación propuesto. Todo ello, como es obvio, alojado en la plataforma de Edmodo, en el grupo correspondiente creado para estos menesteres.

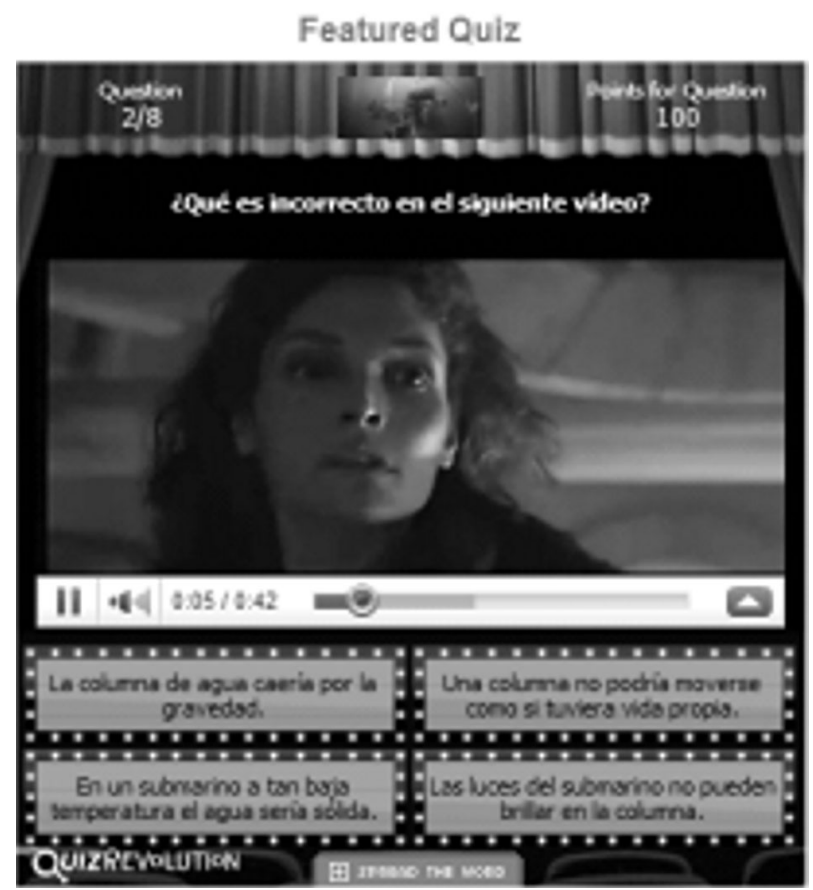

Figura 2. Una de las preguntas del cuestionario multimedia realizado para la película Abyss.

Esta fase se completó con una valoración que efectuó cada grupo de, al menos, tres trabajos diferentes al suyo, con objeto de desarrollar el sentido de la crítica positiva y de la comunicación entre pares.

\section{Evaluación del PROYeCto}

Describiremos en este epígrafe la evaluación efectuada por el profesor, así como los resultados obtenidos de los cuestionarios respondidos por los alumnos.

La evaluación del profesor de la ejecución de este proyecto es muy positiva. Se procede, a continuación, a presentar los puntos fuertes y débiles detectados.

En cuanto a los puntos fuertes del proyecto se pueden resaltar: Se potencian las competencias básicas del aprendizaje, rompe con la rutina tradicional de la enseñanza clásica de la Física y Química de Bachillerato, se incrementa la motivación, el aprendizaje y la capacidad de afrontar situaciones desconocidas por parte del estudiantado, desarrolla habilidades informáticas que favorece la alfabetización digital del estudiantado y la metodología empleada en la realización de este proyecto es extrapolable a otras asignaturas.

Respecto a los puntos débiles se detectan: Una mayor estructuración en la entrega de las tareas para minimizar los colapsos en el uso de la plataforma, una escasa comunicación entre pares y con el profesor y miedo en algunos estudiantes a enfrentarse a situaciones desconocidas (tipo de proyecto y herramientas informáticas (inexploradas).

Con respecto a la evaluación de los alumnos: de los 46 alumnos implicados en el proyecto se recogieron 43 cuestionarios de autoevaluación. El 98,21\% reconoció que le había gustado analizar películas de ciencia ficción, manifestando el 56,61\% que se aprendía Física y el 43,49\% opinaba que se repasaba esta materia. El 100\% de los alumnos manifestaron recomendar el análisis de películas en otros cursos, prefiriendo $4 .^{\circ}$ de ESO. Con respecto a la utilidad de las herramientas Web 2.0, el 100\% declaró que lo habian sido y pensaban que les podian servir para otras asignaturas o futuros niveles. Los alumnos destacaron como herramientas más valoradas Glog/Glogster, MyStudiyo e Issuu. En cuanto a las utilidades descubiertas al usar Edmodo, reseñaron al 100\%, el hecho de evitar la entrega material de las tareas, así como el hecho de poder visualizar los trabajos de otros compañeros. 


\section{BIBLIOGRAFÍA}

Beatriz, Z.; Álvarez, M.; Dans, M.; Paur, A. y Gallego, D. J. (2011): "Capacitación y Gestión del Conocimiento con Herramientas Web 2.0", en Alonso, C. M. y Gallego, D. J. (Eds.): Innovación Tecnológica y Gestión del Talento, editan: UNED, EBS y Anaya, Madrid.

De Clerq, L. (2009): "¿Qué es la Web 2.0?", en Grané, M. y Willem, C. (Eds.): Web 2.0: Nuevas Formas de Aprender y Participar, Editorial Laertes S., Barcelona.

O'Reilly, T. (2005): What is the Web 2.0? Design Patterns and Business Models for the Next Generation of Software, URL: http://oreilly.com/web2/archive/ what-is-web-20.html

Quintanal, F. (2011): "Estilos de Aprendizaje y Rendimiento Académico de Física y Química", en Gallego, D. J. y Alonso, C. (dir.): Innovación y Gestión del Talento, pp. 438-448, Editorial EBS Business School, Cáceres.

Quintanal, F. (2010): "Los Superhéroes Viajan por la Web 2.0.", Revista Pulso, vol. 33, pp. 189-206.

Scopeo (2009): "Formación Web 2.0.", monográfico Scopeo, n. ${ }^{\circ}$ 1, Universidad de Salamanca, Fundación Germán Sánchez Ruipérez. URL: http://scopeo.usal.es/images/documentoscopeo/scopeom001.pdf

Sigalés, C.; Mominó, J. M.; Meneses, J. y Badía, A. (2008): La Integración de Internet en la Educación Escolar Española: Situación Actual y Perspectivas de Futuro, Editorial IN3-UOC, colabora Fundación Telefónica, Barcelona, URL: http://www.fundacion.telefonica. com/es/debateyconocimiento/publicaciones/informe_escuelas/esp/informe.htm 